\title{
Does the Arousal System Contribute to Near-Death and Out-of-Body Experiences? A Summary and Response
}

\author{
Jeffrey Long, M.D. \\ Near-Death Experience Research Foundation \\ (NDERF), Gallup, NM \\ Janice Miner Holden, Ed.D. \\ University of North Texas, Denton, TX
}

\begin{abstract}
In April 2006, an important article appeared in a respected medical journal suggesting a relationship between near-death experiences (NDEs) and the body's arousal system, specifically the phenomenon of rapid eye movement (REM) intrusion. In March 2007, the same authors published another article in the same journal, expanding on the previous article's findings and suggesting a relationship between out-of-body experiences (OBEs) and the arousal system. These articles presented lines of evidence and a study to support the hypothesized relationship. In this paper, we acknowledge the viability and potential value of the hypothesis underlying both articles, but identify substantial weaknesses in both the presented lines of evidence and the studies. We conclude with recommendations for future research that would address the hypothesis and would promote a better overall understanding of NDEs and OBEs.
\end{abstract}

Jeffrey Long, M.D., is a radiation oncologist in private practice in Gallup, NM, founder the Near Death Experience Research Foundation (NDERF), and past director of the International Association for Near-Death Studies (IANDS). Janice Miner Holden, Ed.D., LPC, LMFT, NCC, is Professor of Counseling and Interim Chair of the Department of Counseling, Higher Education, and Early Childhood Education in the College of Education at the University of North Texas in Denton, TX, and former President of LANDS. The authors wish to thank Bruce Greyson, M.D., Allen Katzoff, Allan Botkin, Psy.D., and Michael Sabom, M.D., for their extremely helpful feedback in the preparation of this article. Reprint requests should be addressed to Dr. Long at P.O. Box 2740, Gallup, NM 87305; e-mail: nderf@nderf.org. 
KEY WORDS: near-death experiences; neuroanatomy; REM sleep; sleep disorders; out-of-body experiences.

On April 11, 2006, the medical journal Neurology published an article entitled, "Does the Arousal System Contribute to Near-Death Experience?" written by Kevin Nelson, Michelle Mattingly, Sherman Lee, and Frederick Schmitt. On March 6, 2007, Neurology published a related article entitled, "Out-of-Body Experiences and Arousal," by Nelson, Mattingly, and Schmitt. Neurology is one of the largest and most respected journals devoted to medical research on the human nervous system. Nelson, the first author of both articles, is a nationally known neurologist. The articles received a lot of media coverage, and despite the authors' diligent efforts to present their study's findings in a balanced manner, the media often inflated those findings beyond what Nelson, Mattingly, Lee, and Schmitt claimed, especially after publication of the first article. For these reasons, a clear understanding of the articles is important both to interested members of the public and to the scholarly field of near-death studies.

These articles and their findings were somewhat complex. To help explain them, we first provide some important background information, including a summary of the articles, and then provide our response to them.

The first article, by Nelson, Mattingly, Lee, and Schmitt, addressing a possible relationship between near-death experiences (NDEs) and rapid eye movement (REM) intrusion, was longer and more detailed, and its concepts underlay the conclusions of the second article. That extensive first article included some detailed discussions of neurological pathways, listing 91 references. We will not address the neurological pathway discussions. Instead, we will focus primarily on those points we consider most relevant to an understanding of the relationship between NDEs, out-of-body experiences (OBEs), and REM intrusion, a phenomenon we will explain below.

We want to say at the outset that we respect and appreciate the contribution Nelson, Mattingly, Lee, and Schmitt have made to the field of near-death studies. They raised a plausibie hypothesis. Although we found much to criticize in their methods and findings, we do not want that criticism to be interpreted as lack of regard for them or their efforts. Our heartfelt goal is to encourage future excellent research on NDEs and to encourage open and respectful dialogue. 


\section{Background Information to the Articles}

\section{REM Intrusion}

The major point of the article by Nelson, Mattingly, Lee, and Schmitt was to suggest a connection between NDEs and REM intrusion. Rapid eye movement or REM sleep is a normal phase of sleep that is usually associated with vivid, emotionally intense, bizarre, story-like dreams. During REM, the eyes move around rapidly under closed eyelids, breathing may become irregular, blood pressure may rise, and muscle tone typically is lost to the point of paralysis. Electroencephalogram (EEG) recordings of brain electrical activity during REM sleep are quite similar to EEG recordings during alert wakefulness.

Normally, REM occurs several times throughout the course of a night's sleep. Typically, the first time begins about 90 minutes after a person has fallen asleep, and the last time is the hour or so just before waking up. Sometimes, REM occurs while an individual is awake, usually just as the person is falling asleep or waking up. This phenomenon is called "REM intrusion" into wakefulness. It occurs in two forms (American Psychiatric Association, 2000, p. 610).

One form of REM intrusion is sleep paralysis. In this condition, the person feels awake but cannot move or talk and may feel unable to breathe, although breathing does actually continue. Another form of REM intrusion is sleep-related hallucinations, including those that occur while the person is falling asleep (hypnagogic) and while waking up (hypnopompic). In these situations, the person feels awake but sees or hears things that seem real but that, generally, the person later determines actually were not real:

Most sleep-related hallucinations are visual and incorporate elements of the actual environment. For instance, individuals may describe objects appearing through cracks in the wall or describe objects moving in a picture on the wall. The hallucinations may also be auditory (e.g., hearing intruders in the home) or kinetic (e.g., sensation of flying). (American Psychiatric Association, 2000, p. 610)

These experiences last anywhere from a few seconds to a few minutes, and they end by themselves. They often are terrifying, especially if several elements of the experience occur together, such as feeling awake, hearing intruders in the house, and feeling unable to move or speak (p. 610). 
REM intrusion is, as Nelson, Mattingly, Lee, and Schmitt wrote, "a frequent normal occurrence" (2006, p. 1004; American Psychiatric Association, 2000, p. 610). But if a person has one or both of these types of experience repeatedly, the person qualifies for a sleep disorder diagnosis of narcolepsy (American Psychiatric Association, 2000, p. 615). Another condition that qualifies for a narcolepsy diagnosis is cataplexy. Cataplexy occurs during the course of waking life. While a person feels wide awake and, usually, is feeling a strong emotion, the person experiences a sudden loss of muscle tone on both sides of the body. It feels like an attack of muscle weakness, and it can range from a sagging jaw to a total bodily collapse. As with REM intrusion, in a matter of a few seconds to minutes, the episode ends on its own, with muscle tone and strength restored. The American Psychiatric Association (2000) appeared to distinguish REM intrusion, that is, visual and auditory hallucinations and sleep paralysis, from cataplexy. However, Nelson, Mattingly, Lee, and Schmitt (2006) included cataplexy as a form of REM intrusion.

Because repeated REM intrusion is a symptom of a recognized disorder, some people mistake any occurrence of REM intrusion as a sign of pathology. In fact, a substantial minority of the general population has reported at least one experience of REM intrusion but has not experienced it frequently enough to qualify as a disorder; that is, the experiences do not greatly distress them or impair their ability to function in life (American Psychiatric Association, 2000, p. 610).

\section{Summary of the First Article}

In their introductory paragraph, Nelson, Mattingly, Lee, and Schmitt acknowledged the "powerful transformation of personal beliefs and values" (2006, p. 1003) following an NDE. The authors then stated the "[assumption that] even the most complex psychological process is dependent on brain function" (2006, p. 1003).

In the next section, they defined the near-death experience as "a response to danger" (2006, p. 1003) comprised of several elements from the NDE Scale that Bruce Greyson (1983) designed to distinguish NDEs from nonNDEs and to measure the depth of NDEs. The elements included vivid senses; feelings of peace, joy, and/or cosmic unity; a sense of being out of one's physical body; a sense of an "otherworldly" environment; seeing and/or feeling surrounded by light; a sense of deceased and/or religious spirits; and a sense of 
a border or "point of no return" (Nelson, Mattingly, Lee, and Schmitt, 2006, p. 1004). Nelson, Mattingly, Lee, and Schmitt wrote that "each $\mathrm{NDE}$ is thought unique and contains [elements] in various combinations with no universal element" (2006, p. 1003). They asserted that individual, age, and cultural differences "[suggest] the content of NDE is modified by experience" (2006, p. 1003) and indicated that NDEs are not the automatic, unconscious, forgotten behavior that sometimes occurs in disorders like epilepsy, narcolepsy, and schizophrenia. They summarized how little is known about neurological processes during NDEs, and they discussed the incidence of NDEs.

In the following section, the authors said that the cause of NDEs is currently unknown. They went on to draw comparisons between some features of NDEs and the REM state, specifically the common element of extraordinary light and the occasional element of being "immobilized, alert to the surroundings, and 'aware of being dead"' (2006, p. 1004). They then described some features of REM intrusion and concluded with the speculation that "during crisis, the [loss of muscle tone to the point of paralysis] could reinforce a person's sense of being dead and convey the impression of death to others" (2006, p. 1004).

Nelson, Mattingly, Lee, and Schmitt then described nerve pathways in the brain that are associated with REM sleep. They asserted that "arguments favoring a contribution by REM intrusion to NDE follow five lines of evidence" (2006, p. 1004). First, REM intrusion occurs frequently among normal, healthy people. The authors cited prior research indicating that, in round figures, about 25 percent of people have reported experiencing hallucinations while falling asleep, 5 percent reported sleep paralysis, and 2 percent reported cataplexy. Second, the authors reviewed evidence that REM intrusion underlies other clinical conditions such as narcolepsy, Parkinson's disease, and delirium tremens, the symptoms of withdrawal from severe alcohol addiction. They included reference to an abnormality in a particular area of the brain associated with hallucinatory images that can include "tunnels with a 'golden gate' at one end, angels, and feelings of levitation" (2006, p. 1004). Their point in these first two "lines of evidence" is that because REM intrusion is common and occurs in a variety of clinical conditions, it might be involved in NDEs as well.

In their third "line of evidence," Nelson, Mattingly, Lee, and Schmitt asserted that "NDE elements can be explained by REM intrusion" (2006, p. 1005). Here, the authors compared and contrasted NDE with REM elements. They asserted that some aspects of NDEs, including 
autoscopy, light, visual experience, pleasant feelings, and transcendent qualities, occur in NDEs but are not unique to them and can occur in other clinical conditions including some conditions with an established association with REM intrusion. They concluded that although NDEs and REM "fundamentally differ" (2006, p. 1005) in some ways, REM intrusion occurring at the time of a life-threatening event could account for many elements of NDEs.

Fourth, Nelson, Mattingly, Lee, and Schmitt claimed that danger "undoubtedly" (2006, p. 1005) provokes the arousal of certain nerve pathways that, when aroused, are known to generate REM-associated physiological responses. And, for their fifth "line of evidence," the authors stated that "under apparently similar [physical] conditions, a fraction of cardiac arrest survivors have an NDE" (2006, p. 1006), and they asked whether NDErs had a greater lifetime prevalence of REM intrusion, that is, whether they were more likely than other people to have experienced REM intrusion at some time in their lives.

To try to answer this question, Nelson, Mattingly, Lee, and Schmitt conducted a study involving a survey of an NDE group and a comparison group. For the NDE group, 446 North American adult self-reported NDErs, who had posted their NDEs at the Near-Death Experience Research Foundation (NDERF) web site, were invited by e-mail to participate in a study. Of the 64 who responded, the authors used structured interviews to survey 55 whose NDEs occurred during the authors' definition of a dangerous situation and qualified as NDEs on Greyson's NDE Scale. For the comparison group, they interviewed 55 people "recruited from medical center personnel or their contacts" (2006, p. 1006) who matched the NDEr group by age and sex. In the interview, they asked four questions to assess (1) visual REM intrusion or hallucinations, (2) auditory REM intrusion or hallucinations, (3) atonic REM intrusion or sleep paralysis, and (4) atonic REM intrusion or cataplexy. Respectively, the questions were:

1. Just before falling asleep or just after awakening, have you ever seen things, objects or people that others cannot see?

2. Just before falling asleep or just after awakening, have you ever heard sounds, music or voices that other people cannot hear?

3. Have you ever awakened and found that you were unable to move or felt paralyzed?

4. Have you ever had sudden muscle weakness in your legs or knee buckling? (2006, p. 1007) 
They also calculated "total REM intrusion" by how many survey participants said "yes" to one, two, three, and all four questions.

Except for the cataplexy question, more NDErs said "yes" to each of the questions than did the comparison group members: (1) 42 percent versus 7 percent; (2) 36 percent versus 7 percent; and (3) 46 percent versus 13 percent $(2006, p$. 1007). The number of NDErs who said "yes" to a total of one or more questions also was greater: 60 percent versus 24 percent $(2006$, p. 1007). These differences were statistically significant. The authors concluded that "episodes of REM intrusion appear to be substantially more common in the lifetime of subjects with an NDE. These findings imply that persons with an NDE have an arousal system predisposing to REM intrusion" (2006, p. 1007). Nelson, Mattingly, Lee, and Schmitt ended the article by acknowledging some limitations of the study and identifying some challenges of further research on this topic.

Although they did not state it explicitly, Nelson, Mattingly, Lee, and Schmitt appeared to be hypothesizing a "diathesis-stress model" (Zubin and Spring, 1977) of NDEs. According to this model, some people have a "vulnerable" arousal system (the diathesis), as evidenced by their having experienced REM intrusion, a "glitch" in the sleep/arousal process, at some time in their lives. This model suggests that when such people encounter fear in response to a lifethreatening event (the stress), they are more likely to experience an NDE, which shares some features with REM intrusion. The person need not have shown the symptoms of their vulnerable arousal system prior to the NDE. For example, in the case of posttraumatic stress disorder (PTSD), the symptoms of the person's vulnerable nervous system often appear only after the person has encountered the trauma. However, research indicates that PTSD vulnerability often had a genetic basis that existed prior to the encounter with the trauma and predisposed the person to develop PTSD in response to the encounter (American Psychiatric Association, 2000, p. 466). It should be noted that although the example of PTSD involved a recognized psychological disorder, neither we nor Nelson, Mattingly, Lee, and Schmitt either stated or implied that NDEs themselves indicate disorder. The point here is that the diathesis-stress model of NDEs appears to summarize the basis for Nelson, Mattingly, Lee, and Schmitt's "lines of evidence," their study, and their interpretation of the study findings. 


\section{Summary of the Second Article}

In the second article, Nelson, Mattingly, and Schmitt wrote that "Although not considered REM intrusion, OBE and the REM state have an established relationship that is incompletely understood" (2007, p. 794). This article expanded on their previous survey of an NDE group and comparison group. The occurrence of OBEs during NDEs was determined by participants' responses to the NDE Scale questionnaire that they "clearly left the body and existed outside it." Using this criterion, 56 percent of the NDErs experienced OBEs during their NDEs. Nelson, Mattingly, and Schmitt considered respondents to have had sleep transition OBEs if they answered affirmatively to the survey question: "Just before falling asleep or just after awakening, have you ever had the sense that you are outside of your body and watching yourself?" (2007, p. 794).

Forty-two of the 55 NDErs responded affirmatively to one or both of the survey OBE questions, with 17 experiencing OBEs during their NDE only, 11 during sleep transition only, and 14 during both NDEs and sleep transition. Sleep transition OBEs occurred in 25 NDErs and in three participants in the comparison group; that difference in responses between the two groups was statistically significant. The authors also found that among NDErs, REM intrusion was statistically significantly more common among NDErs with OBEs. They also found that NDErs whose NDEs included OBEs were statistically more likely to have experienced visual and auditory REM intrusion than NDErs whose NDEs did not include OBEs.

Nelson, Mattingly, and Schmitt presented nerve pathways in the brain that may be associated with REM intrusion and/or NDEs. The authors concluded: "This investigation supports OBE as an expression of arousal in NDE and sleep transition. Persons with NDE appear to have an arousal system predisposed to both REM intrusion and OBE" (2007, p. 794).

\section{Our Response to the Articles}

These two articles by Nelson and his colleagues raised some very interesting points and questions regarding a possible relationship between NDEs, OBEs, and the arousal system/REM intrusion. The authors drew conclusions in both articles based on the single survey 
methodology described in the more extensive first article, toward which we will direct most of our comments.

Nelson and his colleagues introduced the first article with accurate descriptions of the typical elements, incidence, and aftereffects of NDEs. However, we take issue with many of their subsequent points throughout both articles. Rather than addressing all these concerns point by point in the same order as the authors presented them in their articles, we will address only our most salient concerns, beginning with those we found most striking and important and proceeding to the more subtle or minor ones.

\section{NDErs' Responses to REM Intrusion Questions}

Our first response to the Nelson, Mattingly, Lee, and Schmitt article is to note that 40 percent of the NDErs said "no" to all four questions designed to assess REM intrusion. Let us assume for the moment that the questions actually assessed REM intrusion and that the NDErs in this study were representative of all NDErs. If 40 percent of NDErs deny ever having experienced a single episode of REM intrusion in their entire lives, the idea that REM intrusion "underlies" and "predisposes" a person to have an NDE when encountering a lifethreatening event seems questionable at best.

In analyzing this first study more closely, we asked what other factors besides REM intrusion could explain the study findings and what other interpretations besides the diathesis-stress model of NDEs could explain the study findings. Our concerns about this study fell into two categories: validity of the questions and composition of the study groups.

\section{Validity of the Questions}

In this section, we will present our arguments for three points. First, when NDErs said "yes" to the survey questions about visual and auditory experiences while falling asleep and waking up, they may have been reporting experiences that did not actually fit the clinical definition of REM intrusion. Such "yes" responses would have artificially inflated the NDErs' reported incidence of "REM intrusion."

Second, when NDErs said "yes" to the survey questions, they may not have been revealing conditions that existed prior to the NDEs, but rather may have been revealing experiences that were aftereffects of 
NDEs. Thus, rather than concluding that NDErs may have had arousal systems that predisposed them to their NDEs and OBEs, it is equally plausible to conclude that they experienced an increase in unusual falling-asleep and waking-up experiences and in OBEs as a result of their NDEs.

Third, when NDErs said "yes" more often to "REM intrusion" and "OBE" questions, they may have been revealing not that they have such experiences more often than others but that, since their NDEs, they have become sensitized to notice and remember unusual experiences, including REM intrusion experiences and OBEs, more often.

One of the most striking issues we found in the Nelson, Mattingly, Lee, and Schmitt study concerned the validity of the questions they used. Validity refers to whether a researcher is getting information on what one thinks one is getting information on. In this first study, for example, Nelson, Mattingly, Lee, and Schmitt defined a lifelong prevalence of visual REM intrusion as an answer of "yes" to the single question, "Just before falling asleep or just after awakening, have you ever seen things, objects or people that others cannot see?" Likewise, other aspects of REM intrusion were defined similarly by a "yes" response to a single survey question. Putting aside for the moment the matter of "lifelong," is a "yes" answer necessarily an indication of REM intrusion? When respondents said "yes," did they have in mind the kind of experience that truly fell into the category of REM intrusion hallucinations?

Kenneth Ring related a relevant case in his 1984 book, Heading Toward Omega. Toward the end of his interview with an elderly woman, she related this experience that had occurred some time after her NDE:

I was awakened one morning with a vision of a woman's forearm holding a box, translucent. And, in the box, there was a beautiful white gardenia. And it wasn't the type of gardenia that we see in this world; it was a spiritual flower. And I heard a voice just as clearly as my own saying, "Take this flower, take this to Mrs. Henry, my mother, and tell her I am always with her."

Now, Dr. Ring, I didn't know any Mrs. Henry, but I had the habit of going to the corner of the cafeteria [at work] every morning for a cup of coffee and I sat at the counter. And I was the only person there except for a woman that sat at the opposite end of the counter. There was no one else there but ourselves. And I hear the waiter say to her, "Would you like another cup of coffee, Mrs. Henry?" And I said [to myself], "Do I dare?" A perfect stranger. A perfect stranger! 
I went up to her afterward and I said, "I beg your pardon. Your name is Mrs. Henry?"

"Yes."

"May I tell you something?"

"Yes."

And I told her what I had heard. She looked at me with stricken eyes, and she said: "A gardenia was my daughter's favorite flower and she has just been killed in an automobile accident." (Ring, 1984, p. 165)

Although we cannot say for sure, it seems quite likely that if this woman had been one of the NDErs in Nelson, Mattingly, Lee, and Schmitt's study, she would have said "yes" to both the visual and the auditory "REM intrusion" questions. But let us compare the content of her experience with the content of falling-asleep and waking-up hallucinations as described by the American Psychiatric Association (2000) and cited above.

First, whereas REM intrusion hallucinations usually "incorporate elements of the actual environment," such as cracks in the wall or a picture on the wall, the NDEr's experience involved elements not in her actual environment: a woman's forearm, a translucent box, a "spiritual" flower, and a woman's voice making reference to someone the NDEr did not know.

Second, whereas REM intrusion hallucinations are bizarre and unrealistic, such as "objects appearing through [those] cracks in the wall or ... objects moving in [that] picture on the wall," the NDEr's experience contained a seemingly coherent and meaningful message, though she was initially mystified as to how to deliver it to the person for whom it apparently was intended.

Third, whereas people typically find REM intrusion experiences frightening, the NDEr neither reported nor implied that she felt frightened during the experience.

Fourth, once people experiencing REM intrusion awaken fully, they generally recognize that the hallucination did not reflect reality: no objects actually in the cracks; objects in the picture actually unmoved from their original positions. By contrast, when the NDEr awoke, she felt perplexed about how she could deliver a message to someone she did not know; her reaction indicated that she considered the message "real" or, at least, potentially real. Furthermore, her subsequent experience confirmed the reality-basis of the message.

It appears that Ring's (1984) interviewee's experience differed fundamentally from REM intrusion hallucinations. He found that 
after their NDEs, experiencers evidenced a substantial increase in experiences like the one his interviewee described, a finding that numerous subsequent researchers have corroborated (Greyson, $2000 \mathrm{~b}$ ). Thus, the possibility exists that when NDErs said "yes" to the "REM intrusion" questions, they were responding at least sometimes on the basis of experiences that were not actually representative of REM intrusion experiences as the latter have been clinically defined. In other words, the researchers thought they were getting responses about REM intrusion when they actually were getting responses based on unusual falling-asleep and waking-up experiences that do not fit the profile of REM intrusion. Because the authors did not record NDErs' narratives that may have accompanied their "yes" responses to the survey questions and might have clarified the actual nature of their experiences, the entire validity of the visual and auditory REM intrusion responses comes into serious doubt. The relatively high percentage of NDErs saying "yes" to these questions may not actually reflect REM intrusion at all.

Indeed, from the wording of the study questions, "Have you ever ...," it is impossible to tell whether the NDEr answering "yes" was referring to one or more experiences before their NDE, after it, or both. One of us (J. L.) determined that NDErs share their experiences at the NDERF web site an average of about 15 years after their NDEs, so responses could very well have reflected post-NDE REM intrusion experiences. The diathesis-stress model provides one plausible interpretation of the study results. However, the questions and their results do not rule out what we will call a "nondiathesis-stress" model, in which changes after an NDE do not reflect a predisposition.

For example, imagine a study in which two groups are asked, "Have you ever experienced a broken bone?" One group consists of people who have been in a car-crushing accident, and the comparison group consists of people who have never been in an accident. The people in the first group are almost certainly going to say "yes" in statistically significantly greater numbers. But it would not make sense to conclude only that the people in the first group had a preexisting proneness to bone breaks. Sometimes the stressor is so powerful that it, rather than any presumed predisposition, is the cause of the effect. By analogy, it is quite possible that, in the aftermath of their NDEs and triggered by their NDEs, people have an increase in unusual falling-asleep and waking-up experiences that may or may not represent REM intrusion. The wording of the study questions, along 
with the entire design of the study, did not rule out the latter interpretation and did not provide specific support for the diathesisstress model.

Along these same lines, the authors found that the higher an NDEr's score on Greyson's NDE Scale, indicating a deeper NDE, the more likely he or she was to say "yes" to the questions about visual and auditory hallucinations. They interpreted this finding as support for the relationship between REM intrusion and NDEs. But given the previous points, an equally plausible interpretation is that "deep" NDErs, whose NDEs were deeper for unknown reasons rather than because of a predisposition of some sort, were more likely to show the aftereffect of having, noticing, and reporting nonordinary visual and auditory experiences around falling asleep and waking up that do not reflect REM intrusion as it is clinically defined. Nelson, Mattingly, Lee, and Schmitt's research method did not rule out this very real possibility.

Given all the points above, Nelson, Mattingly, Lee, and Schmitt's interpretations that "episodes of REM intrusion appear to be substantially more common in the lifetime of subjects with an NDE" and that "these findings imply that persons with an NDE have an arousal system predisposing to REM intrusion" (2006, p. 1007) appear to be overstatements. It is important to note that in the conclusion of the article, the authors stated that REM intrusion may (emphasis added) underlie some of the subjective experiences of NDE and fainting. They acknowledged that their study was not conclusive, and they even avoided using the term "highly suggestive" with regard to the possibility that REM intrusion accounts for some aspects of NDEs. It is a basic tenet of science that retrospective studies, such as this one, are designed to generate hypotheses and cannot be conclusive. In their conclusion, Nelson, Mattingly, Lee, and Schmitt clearly honored this basic principle, though even the degree of their tentativeness may not have been strong enough, given the concerns we have noted.

\section{Composition of Participant Groups}

In conducting a comparison study, one of the cardinal principles of science is to "isolate the independent variable." This phrase means that, whenever possible, the two groups being compared should be exactly the same except for the one variable under investigation. In this study, that variable was a reported NDE. 
The authors followed this cardinal principle by choosing comparison participants who were the same age and sex as the NDE participants. However, they deviated from the principle by choosing medical personnel and their contacts to comprise the comparison group. An ideal comparison group would have been made up of people who had been through life-threatening events comparable to the NDErs'; who matched the NDErs on age, sex, and other identifying aspects such as culture; and who were willing to report their experiences on a public web site, but who had not had an NDE. Consequently, any difference in responses between the two groups in the Nelson, Mattingly, Lee, and Schmitt study might be related to other factors besides an NDE. In this section, we will present our arguments for four points.

First, because of the composition of the comparison group, it is very possible that anyone who survived a life-threatening event, even without an NDE, might report a higher rate of unusual falling-asleep and waking-up experiences. Such findings would provide no evidence of a connection between REM intrusion and NDEs and, thus, no support for the hypothesis that REM intrusion underlies NDEs.

Second, the comparison group in the study, composed of medical personnel and their contacts, may have said "yes" to survey questions at an unusually low rate because they recognized pathological implications in the questions and wanted to avoid those implications. Such an unusually low rate would have artificially increased the difference between the NDEr and comparison group responses, indicating that the groups were more different regarding "REM intrusion" than they actually were.

Third, the NDEr group may have said "yes" to the survey questions more often because they are a particular subset of NDErs who are more inclined to be aware of, notice, and report publicly their unusual experiences. Such an unusually high rate would have artificially increased the difference between the $\mathrm{NDEr}$ and comparison group responses, indicating that the groups were more different regarding "REM intrusion" than they actually were.

Fourth, the researchers may have eliminated some potential participants from the study who might have reduced the difference between the responses of the NDE and comparison groups.

The medical personnel and contacts who participated in the study were less likely to have experienced a prior acute episode of danger comparable to what NDErs had experienced. Thus, the possibility remains that anyone who has been through such a life-threatening 
situation, even without an NDE, would respond to the study survey questions as the NDErs did. If this were the case, the specific connection would have been with "REM intrusion" and survival of a life-threatening situation, not "REM intrusion" and NDEs. It is plausible that any such survival might affect the arousal system; indeed, one common aspect of PTSD is "persistent symptoms of increased arousal" (American Psychiatric Association, 2000, p. 463). Although REM intrusion is not specifically mentioned as one of those symptoms of arousal, it may be an actual but as-yet-unrecognized manifestation in PTSD. In fact, Allan Botkin, who has specialized for 20 years in the diagnosis and treatment of PTSD, has observed a much higher incidence of REM intrusion with PTSD (A. Botkin, personal communication, May 15, 2006). His observation was recently confirmed by researchers who found, among Cambodian refugees attending an American psychiatric clinic, a significantly higher incidence of sleep paralysis among those with PTSD compared to those without it (Hinton, Pich, Chhean, Pollack, and McNally, 2005). These patients' sleep paralysis experiences usually included the other feature of REM intrusion, visual falling-asleep and waking-up hallucinations. The point here is that without knowing how acutely endangered nonNDErs would respond to the study questions, any speculation about a specific connection between NDEs and the arousal system/"REM intrusion" must be tentative at best.

Another unfortunate possibility is that the profound differences between the NDE and comparison groups may have occurred, at least in part, because the researchers specifically used medical personnel or their contacts for the comparison group. Medical center personnel would undoubtedly be more likely than the general public to recognize the pathological implications of the interview questions, that saying "yes" might indicate a "disorder." Despite assurances of confidentiality, for a variety of reasons including to protect their reputations and employment, they may have been less willing to say "yes" to the survey questions even if they had had the experience that the question addressed.

This argument seems to be supported by the study survey results about visual and auditory "REM intrusion" experiences. Only 7 percent of the comparison group said "yes" to these two questions. The Nelson, Mattingly, Lee, and Schmitt article references indicated a prevalence of sleep-related hallucinations in 19 percent, and specifically falling-asleep hallucinations in 24 to 28 percent, of the 
general population, figures consistent with our review of other pertinent literature on these topics (Cheyne, Newby-Clark, and Rueffer, 1999; Fukuda, Ogilvie, Chilcott, Vendittelli, and Takeuchi, 1998). The American Psychiatric Association (2000, p. 610) indicated an overall prevalence of 10 to 15 percent. Thus, even compared to the most conservative estimate, the comparison group's 7 percent response seems low, reinforcing our concern that those respondents may have been unwilling to respond affirmatively to the interview questions.

The evidence regarding sleep paralysis is weaker. Nelson, Mattingly, Lee, and Schmitt cited references indicating that about 6 percent of the general population have reported at least one experience of sleep paralysis. Recent surveys yield widely varying estimates ranging from 2.3 percent to 40 percent (Cheyne, 2005). The American Psychiatric Association (APA) stated that "40\%-50\% of normal sleepers report having had isolated episodes of sleep paralysis at least once during their lifetime" (2000, p. 610) In the Nelson, Mattingly, Lee, and Schmitt study, 46 percent of NDErs reported at least one experience of sleep paralysis, exactly within the norm stated by the APA and somewhat more than the highest range estimated from recent surveys. By contrast, only 13 percent of the comparison group indicated at least one lifelong experience of sleep paralysis. Although this incidence falls within the lowest estimates of recent surveys, it falls well below the norm stated by the APA and somewhat below the mid-range of the estimates from the most recent surveys. These findings are less clear than those on visual and auditory experiences. Nevertheless, they suggest at least the possibility that the extreme difference between the NDE and comparison groups may have been artificially inflated by an underreporting of sleep paralysis experiences by the comparison group.

The comparison group in this study may be considered a convenience sample, one that was presumably more easily available than a scientifically "better" group. Researchers often use convenience samples, but when they do, they should state how those samples deviate from the ideal and how they limit the validity of the findings. Nelson, Mattingly, Lee, and Schmitt did not address this limitation. We acknowledge that finding an ideal comparison group would have been more difficult than using the medical personnel and their contacts who were easily at hand. Unfortunately, because the researchers did not use a more ideal group, the comparison group's 
responses may mean something different from what Nelson, Mattingly, Lee, and Schmitt concluded.

Among NDErs, 42 percent said "yes" to the question about visual experiences and 36 percent to the question about auditory experiences, percentages that are higher than would be expected. One factor in this unusually high rate may be the composition of the NDEr group. When one considers that an estimated 4 percent of the adult population of the United States has experienced an NDE (Gallup, 1982); that out of these millions, less than 1,000 elected to share their NDE on the NDERF web site form; and that out of these, less than 100 volunteered to participate in the Nelson, Mattingly, Lee, and Schmitt study, it is plausible that the resulting 64 NDErs who volunteered to participate in the survey are not typical of the millions of NDErs. Specifically, they may have been predisposed to recall and report nonordinary experiences. This predisposition might result in a high rate of "yes" responses to the survey, a rate that does not represent how most NDErs would respond. This problem of the self-selection of respondents is inherent in retrospective research designs, like Nelson, Mattingly, Lee, and Schmitt's, that dominate NDE research.

A final point involves Nelson, Mattingly, Lee, and Schmitt's exclusion of some willing participants from their study. In identifying people to invite to participate in the Nelson, Mattingly, Lee, and Schmitt study, one of us (J. L.) had determined that all those who were invited had experienced a life-threatening event at the time of their NDEs. However, Nelson, Mattingly, Lee, and Schmitt noted that upon initial interviews, they excluded six participants whom they did not consider to have experienced imminent life-threatening events associated with their NDEs. The basis of this difference of opinion is unclear. Nelson, Mattingly, Lee, and Schmitt apparently made no effort to reconcile this difference of opinion, and they did not provide a separate analysis that included the six excluded participants with the other NDE respondents. Although the probability is low that including these six people in the study would have changed the results, that possibility still exists.

In their study, Nelson, Mattingly, Lee, and Schmitt used groups that did not rule out plausible alternative explanations for their findings and that may have provided erroneous support for their explanation of their findings. Considering both the issues of the validity of study questions and the composition of study groups, it seems most prudent to us to conclude from the study that, for 
now, the diathesis-stress model of NDEs is possible but entirely hypothetical.

\section{Response to the Introductory Material and Lines of Evidence in the First Study}

In the first article, Nelson, Mattingly, Lee, and Schmitt (2006) presented introductory material and five lines of evidence to support a connection between NDEs and the arousal system/REM intrusion. In this section, we address three points that, we believe, cast at least question on, if not serious doubt about, the hypothesized connection. We again begin with the point we consider most salient.

\section{Circumstances of REM Intrusion and NDEs}

For REM intrusion to underlie NDEs, all NDEs would need to occur in circumstances in which REM intrusion was possible. In this section, we describe certain circumstances in which NDEs have been reported, and sometimes documented, in which REM intrusion was very likely or clearly absent: first, when the NDEr had no opportunity to experience fight-or-flight; second, when congenitally blind people, who had never experienced vision or rapid eye movements, had NDEs that included vision; third, when the NDE occurred while the experiencer was under the influence of a medication or drug known to suppress REM, including a documented case of REM suppression; and fourth, when the NDE occurred while the experiencer was documented to be in deep coma, without pulse or breathing, when REM was highly unlikely to have occurred.

In the section of the first article entitled "Cardiorespiratory afferents evoke REM intrusion," Nelson, Mattingly, Lee, and Schmitt discussed "fight-or-flight," a normal physiological response to a lifethreatening event. This response involves the perception of serious danger followed by the emotional experience of intense fear, the physical response of faster heart rate and breathing, and the motivational response to freeze, fight, or flee. The authors described extensively the nerve pathways in the brain associated with the fightor-flight response that also are associated with REM intrusion. Their point was to establish that a life-threatening event, such as that associated with an NDE, could trigger fear-related nerve pathways 
associated with REM intrusion, which would support a possible association between NDEs and REM intrusion.

Our response is to note that NDEs may occur as the result of a lifethreatening event that is sudden and unexpected, involving no opportunity to assess an imminent danger and react with fight-orflight (Greyson, 2000b). Examples include an unanticipated blow to the head resulting in immediate unconsciousness as well as cases of illness and surgery in which the NDEr had not been aware that his or her body was in immediate, life-threatening danger. The common occurrence of NDEs in these circumstances argues against a necessary preexisting psychological state, such as fear, for the occurrence of an $\mathrm{NDE}$; argues against a fight-or-flight psychological/physical reaction as necessarily preceding an NDE; and, thus, severely weakens this particular hypothetical link between NDEs and REM or REM intrusion.

Another source of doubt regarding the link between REM intrusion and NDEs comes from individuals born blind from birth who report that in waking life they have never seen anything, not even blackness, and that their dreams contain sensations of touch, sound, smell, and taste, but no sense of sight. Corresponding to these people's subjective absence of dream vision, research has shown that they have no actual rapid eye movement while they dream. Nevertheless, when such people have NDEs, their experiences contain the typical NDE elements, often including sight (Ring and Cooper, 1998, 1999). It is difficult to imagine how, under life-threatening circumstances, even a dysfunctioning arousal system could generate a subjective perception that the person had never experienced and that was, in fact, neurologically impossible. Such cases provide further strong evidence that REM intrusion does not "underlie" NDEs.

A final point involves the common occurrence of NDEs that experiencers reported while they were under the influence of medication known to suppress REM. For example, experiencers have reported that their NDEs occurred during deliberate or accidental barbiturate overdose.

Even more compelling evidence comes from NDEs that reportedly occurred during general anesthesia, a situation in which the brain functioning necessary for REM intrusion should not be possible. One documented case is that of Pam Reynolds who underwent extensive brain surgery to repair an aneurysm (Broome, 2002; Sabom, 1998). For the first hour and a half of her procedure, she was fully 
anesthetized and prepared to the point of the surgeon cutting into her skull bone. The preparation included taping her eyes shut, inserting clicking devices into her ears that were designed to block out all other ambient noise, and making the incision to expose the bone, all the while continuously monitoring her EEG and two other indicators to ensure that her brain was functioning at only the most basic level. Under these circumstances, the EEG pattern shows extremely low activity, quite different than the awake-like pattern of REM. When the surgeon finally turned on the bone saw to cut into her skull, Reynolds's typical and extensive NDE began, during which she reported seeing and hearing events that were later confirmed to be accurate. Her medical records did not indicate any change in her EEG at this point, indicating that her NDE occurred in the absence of any seizure or REM activity and while her arousal system was suppressed by general anesthesia (M. Sabom, personal communication, May 1, 2006).

A final example of an NDE when REM was unlikely to have been occurring involved a man who arrived at hospital with no pulse or breathing. He was medically monitored to have been in deep coma throughout his resuscitation and for some time afterward. Yet when he later regained consciousness, he accurately reported how, during his resuscitation, a nurse had removed this patient's dentures and placed them in a drawer of the emergency room's metal "crash car" prior to intubating the patient (van Lommel, van Wees, Meyers, and Elfferich, 2001, p. 2041).

In summary, NDEs have been widely reported, and sometimes carefully documented, to have occurred when a person has not had the opportunity to experience the fight-or-flight response, when the person was under the influence of medication or drugs known to suppress REM, and/or when the person was deeply unconscious, neither falling asleep, waking up, nor in a REM state. These circumstances provide strong evidence that neither the fight-or-flight response, REM, REM intrusion, nor any phenomenon involving the arousal system are necessary for NDEs to occur, and, thus, cannot be thought to underlie NDEs.

\section{Comparison of Elements of REM Intrusion and NDEs}

Although Nelson, Mattingly, Lee, and Schmitt affirmed that NDEs and REM dreams are fundamentally different, they also supported a possible link between NDEs and REM intrusion by pointing out 
certain elements that seemed to them to be similar. If NDEs were related to REM intrusion, it would be expected that many or most elements of the two phenomena would be very similar. However, in our comparison of the two types of experience, which we based on consultation with several sources (American Psychiatric Association, 2000; Greyson, 2000a, 2000b; Health-cares.net, n.d.; Ring, 1980, 1984; Parnia, Waller, Yeates, and Fenwick, 2001; Stanford University, 1999; Strauch and Meier, 1996; University of Waterloo, n.d.; van Lommel, van Wees, Meyers, and Elfferich; Wikipedia Foundation, Inc., 2006), we found very little similarity. In this section, we discuss our findings in this regard for the elements that Nelson, Mattingly, Lee, and Schmitt identified in the first article: "autoscopy," extraordinary light, a sense of "being dead," visual and auditory hallucinations, and other elements.

Autoscopy. We are concerned with Nelson, Mattingly, Lee, and Schmitt's use of the term "autoscopy" in the context of REM intrusion and NDEs. In an early NDE study, Michael Sabom (1982) used this term. However, shortly thereafter, psychiatrists Glen Gabbard and Stuart Twemlow (1984) clarified that autoscopy is the experience of viewing a visual double of one's body while remaining in the physical body. We found no such experience reported in either the REM intrusion or the NDE literature.

We did find REM intrusion and NDE accounts that included viewing one's physical body from a perspective outside the body, a phenomenon usually termed in the professional literature "out-of-body experience" (OBE). However, OBEs appeared to us to be common in NDEs and much less common in REM intrusion. In fact, in the vast majority of REM intrusion experiences, the person feels "in" one's body, often terrifyingly trapped in one's body while enduring paralysis and/or hallucinations. By contrast, an NDE usually involves the sense of being conscious but no longer associated with one's body, an experience almost always accompanied by feelings of profound peace and well-being. In our collective experience as NDE researchers, we have never encountered an NDE in which a person felt frantically trapped in one's body during the NDE itself. Furthermore, we found that the OBEs associated with REM intrusion were usually accompanied by a fearful loss of control, as compared with the vast majority of OBEs in NDEs being accompanied by peace, well-being, acceptance, and even benign interest. Nelson, Mattingly, and Schmitt concluded the second article, "This investigation supports OBE as an expression 
of arousal in NDE and sleep transition" (2007, p. 794). The substantial differences between the content of OBEs during NDEs and sleep transition REM intrusion cast considerable doubt on this conclusion.

In light of these distinctions alone, we find that NDEs and REM intrusion as discussed in the first article are fundamentally different. Rather than concluding that REM intrusion underlies NDE, we think it equally, if not more, plausible to conclude that NDEs occur in a variety of circumstances, possibly occasionally in association with REM intrusion, but that the two experiences are fundamentally different.

On a related but perhaps less salient note, another of our concerns is that Nelson, Mattingly, Lee, and Schmitt (2006, p. 1005) used the article by Olaf Blanke, Stéphanie Ortigue, Theodor Landis, and Margitta Seeck (2002) to support their statement that "autoscopy is directly produced by" (2006, p. 1005) electrical stimulation of a particular area of the brain. We and Jason MacLurg have already published a paper, in response to the article by Blanke, Ortigue, Landis, and Seeck, explaining that such electrically induced OBEs are quite different from OBEs that occur in NDEs (Holden, Long, and MacLurg, 2006).

Other elements and features. Nelson, Mattingly, Lee, and Schmitt asserted in the first article that REM intrusion experiences and NDEs share the element of unusual light. However, in our review of light as described in REM intrusion and NDEs, we found two important differences: quality and frequency of reports. The light that NDErs report usually has a mystical quality, and reports of such light are extremely frequent in NDEs. By contrast, we did not see the mystical quality described or implied nearly as intensely or frequently in REM intrusion experiences. To us, the quality and frequency of light in these two experiences seems to be quite different. If REM intrusion were a significant contributor to NDEs, we would expect them to be more similar.

Regarding a sense of "being dead," NDErs do, indeed, frequently report that their NDEs included not just a sense of being dead but a realization that they were dead or were in the first phase of death. They almost always report that this realization was accompanied by a sense of peace, even matter-of-fact acceptance, if not benign curiosity. By contrast, REM intrusion experiencers rarely report a sense of being dead but rather a fear they will die, usually in connection with the feeling of being paralyzed in the body and unable 
to breathe. In the rare cases of REM intrusion in which experiencers see their physical bodies from an out-of-body perspective, they sometimes report thinking that they might or must be dead, but they almost never report the conviction of having been dead that is quite common among NDErs.

Regarding visual and auditory hallucinations, both REM intrusion experiencers and the experts who study their experiences agree that many, if not most, of their visual and auditory experiences, such as wall cracks that spawn objects, picture contents that move, or intruders in the house, were not reality-based, but that they were, in fact, hallucinations. By contrast, visual and auditory experiences during NDEs do not fit the profile of hallucinations (Greyson, 2000a). In fact, the literature contains numerous anecdotes (Ring and Lawrence, 1983) and at least two cases involving close monitoring in hospital (Sabom, 1998; van Lommel, van Wees, Meyers, and Elfferich, 2001) of NDEs that included realistic observations that were not knowable to the NDEr through normal sensory channels yet were later confirmed to be accurate.

Indeed, one of us (J. M. H.) has searched the professional near-death literature and contacted several near-death researchers in an explicit attempt to find cases of realistic observations during NDEs that were later confirmed to be inaccurate. Using all sources available to her that were published before Raymond Moody's seminal 1975 book on NDEs and all systematic studies with more than one participant since 1975 , she found 109 cases of allegedly realistic out-of-body perceptions during NDEs that should have been impossible, considering the condition and/or location of the experiencer's physical body. Using the most stringent criterion that a case would be designated inaccurate if even one detail of the account was found not to correspond to consensus reality, she found that only 8 percent of cases involved any inaccuracy at all. Furthermore, 38 percent of the cases involving apparently completely accurate perception were corroborated as accurate by independent, objective sources (Holden, 2006).

Another of us (J. L.) has reviewed approximately 800 NDEs submitted to the NDERF web site, with many hundreds containing realistic observations of events in the immediate physical environment during the NDE while the NDErs apparently were unconscious. More than 98 percent of the realistic observations NDErs described were either completely plausible based on their descriptions, or were confirmed as accurate by the NDEr having later checked the accuracy 
of their NDE observations. Thus, a substantial body of evidence addresses the reality of experiences during REM intrusion and NDEs. These data support the conclusion that most visual and auditory experiences during REM intrusion do fit the profile of hallucinations, whereas the vast majority of such experiences during NDEs do not.

We found several additional differences between the content of REM intrusion and that of NDEs. For example, in NDEs, experiencers often encounter deceased persons whom they can identify, whereas in sleep paralysis or visual and auditory hallucinations, this experience is uncommon. Furthermore, REM intrusion experiences almost never involve the frequent NDE features of a tunnel, a life review, or a decision to return to the body - the latter because, as previously stated, the vast majority of REM intrusion experiences do not include a sense of having left the body. Whereas people in sleep paralysis typically focus on fearful things happening to their bodies or in their physical environments, NDErs typically feel profound peace and wellbeing in the face of unusual things happening to them apart from their physical bodies or physical environments. Finally, REM intrusion experiences seem far more varied in their content than the previously noted consistency of NDE elements. What REM intrusion features are relatively consistently described, such as the sense of being unable to breathe, have not been described in NDEs.

In contrast to Nelson, Mattingly, Lee, and Schmitt, we assert that, despite a few superficial similarities, NDEs are not easily explained by REM intrusion. Our assertion is supported by NDErs' responses to a particular item on the NDERF web site survey: "Following the experience, have you had any other events in your life, medications or substances which reproduced any part of the experience?" Respondents respond "yes," "uncertain," or "no," followed by a "Please explain" text box for a narrative. This question was deliberately worded to encourage as many positive responses as possible. Of 397 respondents who shared their NDEs an average of 16 years after the experience - plenty of time to have subsequent experiences - only 22 percent said "yes." In actuality, the narrative responses frequently addressed experiences both prior to and following their NDEs. Very few suggested REM intrusion; most expanded on a "no" response, in which they described a subsequent NDE or described an experience during meditation or substance abuse. Except for a subsequent NDE during a life-threatening event, virtually no response indicated any life experience that substantially reproduced the entirety of the 
originally reported NDE. Virtually no NDEr described an experience consistent with a REM intrusion associated OBE as reproducing any part of the NDE. The nearly complete absence of NDErs in the NDERF survey to list any REM intrusion experience as having reproduced any part of their NDE further suggests that NDEs and REM intrusion are different experiences.

To summarize, most REM intrusion experiencers have reported relatively brief, frightening paralysis and/or bizarre visual and auditory experiences while they experienced themselves as in the body, experiences that occurred during pre- or post-sleep consciousness or semiconsciousness and not in response to a life-threatening event. The mostly unrealistic visual and/or auditory experiences felt real at the time but were later acknowledged to be unreal. By contrast, NDErs have reported experiences ranging from brief to prolonged, involving elements that comprised a coherent narrative of events while they experienced themselves as out of the body during any of a variety of circumstances, usually life-threatening ones. In many cases, the reports included detailed, ordered, realistic accounts of events in the vicinity of their physical bodies, during which they reported fear only rarely, and after which, upon regaining consciousness, the NDErs were typically adamant that their experience was real, a claim bolstered frequently by subsequent corroboration of their observation of events in the immediate physical environment at the time of their NDEs.

For NDEs to be attributed to REM intrusion, we would expect the two subjective experiences to be substantially similar. We find that they are not. OBEs occurring during NDEs and during REM intrusion appear to be quite different experiences. We also find another striking dissimilarity: the profound aftereffects of NDEs contrasted with the virtual absence of reports of life-changing aftereffects from REM intrusion experiences. If REM intrusion underlay NDEs, we would expect at least somewhat similar aftermaths of the two experiences.

\section{NDE Content Comparison by Age and Culture}

In an early section of the article entitled "What is NDE?" Nelson, Mattingly, Lee, and Schmitt suggested that the content of NDEs is modified by age at the time of the NDE and cultural background of the NDEr. Although the Nelson, Mattingly, Lee, and Schmitt article comments were brief regarding these points, we consider them 
important to address. In this section, we will present our arguments that (1) like REM dreams, NDEs show surface differences based on experiencers' differing ages, cultures, and life experiences; and (2) unlike REM dreams, NDEs show basic consistency despite experiencers' differing ages and life experiences and may show basic consistency despite differing cultures.

Age. The possible influence of age on NDE content has not been well studied. To support their assertion, Nelson, Mattingly, Lee, and Schmitt cited one article by Melvin Morse, Paul Castillo, David Venecia, Jerrold Milstein, and Donald Tyler (1984). In that study, the researchers interviewed only 11 children ranging from 3 to 16 years old. Although the authors noted an absence of life review, time alteration, worldly detachment, or transcendent feelings in the childhood NDE accounts, they concluded that "the elements of NDEs reported are similar to those previously described in adults" (Morse, Castillo, Venecia, Milstein, and Tyler, 1984, p. 1110). Whereas those authors appropriately limited their conclusion to the "NDEs reported" in their study, Nelson, Mattingly, Lee, and Schmitt inappropriately drew a sweeping conclusion about the comparative content of children's and adults' NDEs. Curiously, Nelson, Mattingly, Lee, and Schmitt's conclusion contradicted the original conclusion by Morse, Castillo, Venecia, Milstein, and Tyler. In addition, subsequent case studies of children's NDEs have included life review and worldly detachment (Bonenfant, 2004).

We have found only one other published study that directly compared the elements of children's and adults' NDEs. Nancy Bush (1983) studied 17 pediatric NDEs and found adult and pediatric NDEs comparable. Again, contrary to Nelson, Mattingly, Lee, and Schmitt's conclusion, the results of this study supported more similarity than difference in the content of children's and adults' NDEs.

In a review of professional literature on children's NDEs, Pamela Kircher, Jan Holden, P. M. H. Atwater, Morse, and the IANDS Board of Directors concluded that "the same features reported by adult NDErs have been reported also by child NDErs" (2003, p. 2). However, they described a few differences. Children's NDEs tend to include fewer elements; to be more concrete and less complex; and to include more deceased pets or other animals, relatives whom the child does not recognize at the time (but might later recognize in old family photos the child had not previously seen), and, rarely, living people (2003, p. 2). 
A way to understand the findings about comparisons of children's and adults' NDEs is to use the concept of deep and surface structures (Chomsky, 1969; Wilber, 2000). For example, the generic concept of "a face" is a deep structure: it includes certain facial structures in certain relative positions. But any specific face, while reflecting that deep structure, will also reflect surface structure, the expression of specific genetics, age, and culture: size, shape, skin color, and adornment of the particular facial features. Noam Chomsky originally developed the concept of deep and surface structures to explain both the superficial diversity and the underlying uniformity of grammar worldwide. The concept has been applied since to many other phenomena. We think it also is applicable to NDEs, which contain certain elements that tend to occur in a certain order, such as the features identified in the Greyson's NDE Scale, but that can vary in their specifics based on a variety of experiencer characteristics.

Most relevant to this article is the point that no deep structure has been hypothesized for REM dreams. Such dreams are not comprised of variations on certain elements tending to occur in a certain order; they do not follow a basic prototype like the one NDEs appear to follow. It seems to us counterintuitive to think that the basically unstructured phenomenon of REM dreams would constitute the foundation for the deeply structured phenomenon of NDEs. In Ken Wilber's terms, to think in such terms is to make a category error: REM dreams are predominantly the prerational workings of the prepersonal mind, whereas NDEs are the transrational workings of the transpersonal mind (Wilber, 2000). They are valuable but different sources of information, and to reduce a transrational phenomenon to a prerational one is to lose its essential quality.

Culture. Possible cultural influence on the content of NDEs has also not been well studied. The only reference in the Nelson, Mattingly, Lee, and Schmitt article regarding this subject was a study of 16 NDEs from India (Pasricha and Stevenson, 1986). In that study, the authors' primary purpose in visiting India was to study reincarnation. They said they learned about the NDE cases incidentally, mostly from onlookers to their work who would typically ask, "Are you people also interested in persons who die and recover?" Thus, it is quite possible the NDEs they studied did not represent Indian NDEs as a whole. The authors presented four narratives typical of the NDE accounts they encountered. These narratives were clearly quite different from typical Western NDEs, and the authors addressed the specifics of 
these differences. However, it is important to note that of the 16 NDE accounts they collected, six were reported not by the NDEr personally but by others familiar with the account. These six secondhand accounts by nonNDErs may have involved inaccuracy or distortion, including a tendency to recall and share elements of the NDE that were consistent with their own prior cultural beliefs. In concluding the article, the authors

caution against accepting this observation as adequate evidence that the cases derive only from culture-bound beliefs. Some differences may derive from the effects of a person's beliefs on what actually does happen after death, and some different features may, on closer examination, be found to be basically similar in nature if not in detail. (Pasricha and Stevenson, 1986, p. 165)

Despite this caution, Nelson, Mattingly, Lee, and Schmitt asserted that "cultural heterogeneity [of NDEs] suggests the content of NDE is modified by experience" (p. 1003) without addressing the possibility of some crossculturally consistent features.

Unfortunately, only a scant additional literature has addressed the possible relationship between cultural beliefs and NDE content. Of the few existing reports, many included only small numbers of nonWestern NDEs (usually less than five), used historical accounts rather than first-person interviews, and/or contained accounts without convincing documentation of a life-threatening event at the time of the experience.

However, researchers have conducted several reasonably welldocumented studies of NDEs from nonWestern civilization. The largest of these involved 32 NDErs who survived a 1976 earthquake in China (Zhi-ying and Jian-xun, 1992). The authors compared the frequency of NDE elements between the Chinese NDErs and previously published data from American NDErs. They concluded that "these differences suggest that the components, sequences, and types of NDE might differ with race, religion, psychological and cultural background, and kind of near-death event" (Zhi-ying and Jian-xun, 1992, p. 39). A closer review of the data presented in that article reveals the typical spectrum of NDE elements but reported in a smaller percentage of Chinese NDEs compared to American NDEs. Unfortunately, the authors included no narratives of representative Chinese NDEs. We were unable to discern from this study whether possible differences between Chinese and American NDEs are major or minor in degree. 
To further investigate a comparison of crosscultural NDE content, one of us (J. L.) perused 14 nonWestern NDEs submitted to the NDERF web site. He noted striking similarity of the basic elements of these nonWestern experiences with Western ones (details including Internet webpages on which these NDE accounts appear are available from the author).

The data on crosscultural NDEs are meager and mixed. However, we find that these data tend to indicate surface differences and underlying similarity of NDEs across cultures. Once again, the deep/ surface structure model may be a useful one in understanding the relationship between culture and NDEs.

Life experience. Two studies have addressed the effect of experience on NDE content by examining how much NDErs might have known about NDEs at the time of their experience. Both studies compared NDE elements before and after 1975 when NDEs became widely known. The authors of one study (Athappilly, Greyson, and Stevenson, 2006) found that NDEs reported after 1975 were more likely to contain the element of the tunnel than those reported before 1975. However, the incidence of 14 other NDE elements did not differ before and after 1975. In the other study (Long and Long, 2003) the incidence did not differ for any of the elements the researchers examined. These findings indicate that knowledge of NDEs does not change the type or incidence of elements reported in NDEs.

The NDERF web site questionnaire for NDErs asks for various elements of the respondent's experience and gives 13 check-box options and an additional option of "None of the above." The 13 options include 12 that refer to the elements of the NDE and one indicating "Features consistent with your beliefs at the time." Out of 465 NDErs who completed the survey, 18 percent indicated the latter option, whereas 19 to 76 percent indicated each of the other 12 options. This finding indicates that a substantial majority of NDErs themselves do not perceive that their NDEs conformed to their preexisting beliefs.

Virtually all NDE investigators agree that the similarities between NDEs are far more impressive than their differences. In Wilber's (2000) terms, they affirm the "deep structure" of NDEs that is not lost in their surface structure variations across individual developmental level, culture, and life experience. We see more consistency in NDEs than in any other relatively common human experiences involving altered consciousness, such as REM dreams, hallucinations, posttraumatic nonNDE experiences, or psychotic experiences. We believe 
that the seemingly relative consistency of NDEs supports the idea that they are qualitatively different than REM intrusion and cannot be explained by it.

\section{Summary and Conclusion}

Nelson, Mattingly, Lee, and Schmitt concluded their first article by stating that "REM intrusion may underlie some of the subjective experiences of NDE and [fainting]" (p. 1008). To summarize our points that cast doubt on even this tentative conclusion:

First, 40 percent of the NDErs said "no" to all of the alleged REM intrusion questions, a substantial minority that alone brings into question the authors' conclusion that REM intrusion may underlie NDEs.

Second, when NDErs said "yes" to visual and auditory experience questions, they may have been indicating experiences that do not actually represent REM intrusion, creating the impression of greater alleged REM intrusion where it may not actually have existed.

Third, the questions that Nelson, Mattingly, Lee, and Schmitt used did not differentiate when the alleged REM intrusion experiences occurred relative to when the NDEs occurred; without this differentiation, it is unknown whether alleged REM intrusion underlies and predisposes a person to an NDE or whether it is an aftereffect precipitated by an NDE.

Fourth, the comparison group members they used in their study were much less likely to have survived situations of acute danger comparable to what NDErs had experienced. NDErs' greater proportion of "yes" responses to survey questions may have been related to their having survived a life-threatening event, not their having had an NDE, which would have indicated no specific relationship between alleged REM intrusion and NDEs.

Fifth, the large difference in responses between the NDE and comparison groups may have been artificially increased by NDErs who were more prone to say "yes" to the survey questions, comparison group members who were less prone to say "yes," and the exclusion of some willing participants from the study whose answers might have reduced the difference.

Sixth, NDEs that occur in the absence of the fight-or-flight response that may activate REM, in persons with congenital blindness, and in person under the influence of drugs known to suppress REM, all discredit the argument that REM underlies NDEs. 
Seventh, although the surface content of both REM dreams and NDEs vary with the experiencer's age, culture, and prior life experience, REM dreams apparently do not have a consistent deep structure, whereas NDEs apparently do - a fundamental difference that argues against a REM intrusion basis to NDEs.

Eighth, despite superficial similarities between some elements of REM and REM intrusion, on the one hand, and NDEs on the other, the differences between these experiences appear to outweigh substantially the similarities.

In their conclusion, the authors indicated that prospective study should be undertaken to investigate further the possible relationship between REM intrusion and NDEs. We agree wholeheartedly. Such a study might involve elements such as: (1) developing and then using an instrument and/or an interview protocol with established validity and reliability that assesses if, when, and how often in their lifetimes respondents have experienced REM intrusion and OBEs; (2) recording the narratives of the respondents' experiences associated with answering "yes" to REM intrusion and OBE questions; and (3) using a prospective research design that would yield appropriate comparison groups, such as assessing a large sample of participants experiencing a near-death event before it, immediately after it, and at some established follow-up point in the future.

As an ideal example, a study could take place in a few hospitals for a period of time, such as a year, in which all patients who had never experienced cardiac arrest would complete a valid and reliable REMintrusion and OBE history survey upon entering the hospital, again upon discharge, and again after one year. For those patients who experienced cardiac arrest during the hospital stay, researchers could conduct an interview to determine the presence or absence of NDEs and, when present, administer the Greyson NDE Scale to confirm the NDE and measure its depth. This process would create three groups of former patients: those who had not experienced cardiac arrest, those who had but did not report an NDE, and those who had and also reported an NDE that met the NDE Scale criterion. Researchers could use participants from the two nonNDE groups matched to the NDE group on demographic characteristics to comprise the comparison groups. The researchers would then have quantitative data about the incidence and frequency of REM intrusion and out-of-body experiences before, immediately after, and at some more distant future time following cardiac arrests with NDEs, cardiac arrests without NDEs, 
and nonarrests. The researchers could enrich their findings with qualitative data, interviews in which they solicited narratives regarding OBEs and REM intrusion or other unusual falling-asleep or waking-up experiences, from their participants. It would also be helpful for NDErs to compare and contrast elements of their NDE with any experiences of REM intrusion they may have had.

Nelson, Mattingly, Lee, and Schmitt's assumption that "even the most complex psychological process is dependent on brain function" (p. 1003) is, indeed, as Peter Fenwick (2004) and Pim van Lommel (2004) have reminded us, an unproven assumption (Broome, 2002). Rather than the brain being the producer of consciousness, it may instead be a receiver and transmitter of consciousness that can function independent of the brain. From the latter perspective, even brain functions that are shown consistently to precede or coexist with certain experiences cannot be said to "cause" them, because the "cause" may be sources beyond the brain that the brain is merely able to mediate. In this sense, the most that can be said is that some brain functions correlate with some experiences. Specifically, REM intrusion might correlate with NDEs, but, in our opinion, Nelson, Mattingly, Lee, and Schmitt did not provide credible support for even this more conservative conceptualization.

Thus far, scholars have hypothesized more than 20 different biological and/or psychological causes of NDEs, yet not one of these explanations, nor any combination, has yet been widely accepted. Researching potential correlates of NDEs will continue to be challenging. In this article, we have named only a few methodological challenges. In addition to these challenges, researchers with expertise on possible physiological and psychological correlates of NDEs may have relatively little experience with NDEs. Conversely, researchers knowledgeable about NDEs may have relatively little expertise in the areas of physiological or psychological science necessary to understand proposed correlates of NDEs. In the future, it would be helpful for researchers with different fields of knowledge to collaborate on studies such as the one conducted by Nelson, Mattingly, Lee, and Schmitt. The pooling of knowledge in such a collaborative venture would almost certainly help greatly in advancing a collective understanding of NDEs.

Nelson, Mattingly, Lee, and Schmitt did conclude that, regardless of how NDEs arise, they may have intense personal meaning. Indeed, many NDErs have found their experiences to be profoundly meaning- 
ful. Consequently, contrary to Nelson, Mattingly, Lee, and Schmitt's assumption that NDEs have a "neurophysiological basis" (p. 1003) or "physiological basis" (p. 1004), most NDErs believe their experiences involved elements that were spiritual in origin and, thus, cannot be explained in purely physiological/medical terms. In Wilber's (2000) terms, NDEs may be transpersonal experiences that cannot be reduced to exclusively biological or prepersonal causes.

We believe that a spiritual/transpersonal hypothesis of NDEs might be supported in a number of ways. One important example is research on veridical perception, the situations in which inexplicably accurate perceptions during NDEs have occurred at a time of unconsciousness, cardiopulmonary arrest, and even an absence of measurable brain activity (Holden and Joesten, 1990; Parnia, Waller, Yeates, and Fenwick, 2001; Ring and Lawrence, 1993). Another approach is more indirect: by identifying all reasonable alternative biological and medical explanations, carefully investigating them, and, where little or no support is found, dismissing them.

A final possibility for investigating a spiritual/transpersonal hypothesis of NDEs would be to follow up on previous research that examined consistencies among NDEs. That research began with the assumption of consistency and found it: an out-of-body experience, passing through a tunnel, seeing a light, feeling intense emotions, encountering deceased relatives, entry into an unworldly realm, a life review, a decision to return, and many other less well known elements. Researchers in a future study would begin with no assumption of consistency and formally study a large number of NDE spontaneous narratives, analyzing the degree of both consistency and inconsistency of all aspects of the content, including the spiritual/transpersonal content. If the consistency found in previous research were confirmed, this finding would strongly suggest that physiological and psychological processes alone are not the sole cause of NDEs, and would further validate NDEs as experiences of great significance and meaning regardless of their possible physiological or psychological correlates.

In this paper, we have provided some evidence that supports a spiritual/transpersonal understanding of NDEs, and additional evidence exists. The totality of that evidence is convincing to many critical thinkers. Much additional research and discussion will be necessary for the wider medical and scientific community and the world at large to reach consensus on the biological/medical or spiritual/transpersonal correlates of NDE. In our opinion, these two 
studies by Nelson and his colleagues are important early steps in this difficult but necessary journey toward truly understanding NDEs.

\section{References}

American Psychiatric Association (2000). Diagnostic and statistical manual of mental disorders ( $4^{\text {th }}$ ed., text revision). Washington, DC: Author.

Athappilly, G., Greyson, B., and Stevenson, I. (2006). Do prevailing societal models influence reports of near-death experiences? A comparison of accounts reported before and after 1975. Journal of Nervous and Mental Disease, 194, 218-222.

Blanke, O., Ortigue, S., Landis, T., and Seeck, M. (2002). Stimulating illusory own-body perceptions. Nature, 419, 269-270.

Bonenfant, R. (2001). A child's encounter with the devil: An unusual near-death experience with both blissful and frightening elements. Journal of Near-Death Studies, 20, 87-101.

Broome, K. (Producer). (2002). The day I died [Film]. Glasgow, Scotland: British Broadcasting Corporation.

Bush, N. (1983). The near-death experience in children: Shades of the prison-house reopening. Anabiosis: The Journal of Near-Death Studies, 3, 177-193.

Cheyne, J. (2005). Sleep paralysis episode frequency and number, types, and structure of associated hallucinations. Journal of Sleep Research, 14, 319-324.

Cheyne, J., Newby-Clark, I., and Rueffer, S. (1999). Relations among hypnagogic and hypnopompic experiences associated with sleep paralysis. Journal of Sleep Research, 8, 313-317.

Chomsky, N. (1969). Deep structure, surface structure, and semantic interpretation. Bloomington, IN: Indiana University Linguistics Club.

Fenwick, P. (2004). Science and spirituality: A challenge for the $21^{\text {st }}$ century. Journal of Near-Death Studies, 23, 131-157.

Fukuda, K., Ogilvie, R., Chilcott, L., Vendittelli, A.-M., and Takeuchi, T. (1998). The prevalence of sleep paralysis among Canadian and Japanese college students. Dreaming, 8, 59-66.

Gallup, G., and Proctor, W. (1982). Adventures in immortality: A look beyond the threshold of death. New York, NY: McGraw-Hill.

Greyson, B. (1983). The near-death experience scale: Construction, reliability, and validity. Journal of Nervous and Mental Disease, 171, 369-375.

Greyson, B. (2000a). Dissociation in people who have near-death experiences: Out of their bodies or out of their minds? Lancet, 355, 460-463.

Greyson, B. (2000b). Near-death experiences. In E. Cardeña, S. J. Lynn, and S. Krippner (eds.), Varieties of anomalous experience: Examining the scientific evidence (pp. 315-352). Washington, DC: American Psychological Association.

Health-cares.net [n.d.]. What is hypnagogic hallucination? Retrieved May 1, 2006, from http://neurology.health-cares.net/hypnagogic-hallucination.php

Hinton, D. E., Pich, V., Chhean, D., Pollack, M. H., and McNally, R. J. (2005). Sleep paralysis among Cambodian refugees: Associated with PTSD diagnosis and severity. Depression and Anxiety, 22, 47-51.

Holden, J. M. (2006). Veridical perception in near-death experiences. Paper presented at the 2006 North American Conference of the International Association for Near-Death Studies, "Near-Death Experiences: 30 Years of Research," Houston, TX, October 25, 2006. (Available as videotape from the International Association for Near-Death Studies, P. O. Box 502, East Windsor Hill, CT 06028-0502; http://www.iands.org/ shoppingcart/index.php?main_page $=$ index\&cPath $=48 \_49$ ) 
Holden, J. M., and Joesten, L. (1990). Near-death veridicality research in the hospital setting: Problems and promise. Journal of Near-Death Studies, 9, 45-54.

Holden, J. M., Long, J., and MacLurg, J. (2006). Out-of-body experiences: All in the brain? Journal of Near-Death Studies, 25, 99-107.

Kircher, P. M., Holden, J., Atwater, P. M. H., Morse, M., and the IANDS Board of Directors (2003). Children's near-death experiences [Brochure]. East Windsor Hill, CT: International Association for Near-Death Studies.

Long, J., and Long, J. (2003). A comparison of NDEs occurring before and after 1975: Results from a web survey of near death experiencers. Journal of Near-Death Studies, 22, 21-32.

Moody, R. (1975). Life after life. Covington, GA: Mockingbird Books.

Morse, M., Castillo, P., Venecia, D., Milstein, J., and Tyler, D. C. (1984). Childhood neardeath experiences. American Journal of Diseases of Children, 140, 1110-1114.

Nelson, K., Mattingly, M., Lee, S. A., and Schmitt, F. A. (2006). Does the arousal system contribute to near death experience? Neurology, 66, 1003-1009.

Nelson, L., Mattingly, M., and Schmitt, F. A. (2007). Out-of-body experiences and arousal. Neurology, 68, 794-795.

Parnia, S., Waller, D. G., Yeates, R., and Fenwick, P. (2001). A qualitative and quantitative study of the incidence, features and aetiology of near-death experiences in cardiac arrest survivors. Resuscitation, 48, 149-156.

Pasricha, S., and Stevenson, I. (1986). Near-death experiences in India: A preliminary report. Journal of Nervous and Mental Disease, 174, 165-170.

Ring, K. (1980). Life at death: A scientific investigation of the near-death experience. New York, NY: Coward, McCann, and Geoghegan.

Ring, K. (1984). Heading toward omega: In search of the meaning of the near-death experience. New York, NY: William Morrow.

Ring, K., and Cooper, S. (1998). Near-death and out-of-body experiences in the blind: A study of apparent eyeless vision. Journal of Near-Death Studies, 16, 101-147.

Ring, K., and Cooper, S. (1999). Mindsight: Near-death and out-of-body experiences in the blind. Palo Alto, CA: Institute of Transpersonal Psychology.

Ring, K., and Lawrence, M. (1993). Further evidence for veridical perception during near-death experiences. Journal of Near-Death Studies, 11, 223-229.

Sabom, M. (1998). Light and death: One doctor's fascinating account of near-death experiences. Grand Rapids, MI: Zondervan.

Stanford University. (1999). Sleep paralysis. Retrieved May 1, 2006, from http:// www.stanford.edu/ dement/paralysis.html

Strauch, I., and Meier, B. (1996). In search of dreams: Results of experimental dream research. Albany, NY: State University of New York Press.

University of Waterloo [n.d.]. Sleep paralysis and associated hypnagogic and hypnopompic experiences. Retrieved May 1, 2006, from http://watarts.uwaterloo. ca/-acheyne/S_P.html\#tabcon

van Lommel, P. (2004). About the continuity of our consciousness. Advances in Experimental Medicine and Biology, 550, 115-132.

van Lommel, P., van Wees, R., Meyers, V., and Elfferich, I. (2001). Near-death experience in survivors of cardiac arrest: a prospective study in the Netherlands. Lancet, 358, 2039-2045.

Wilber, K. (2000). Integral psychology. In K. Wilber (ed.), The collected works of Ken Wilber (vol. 4). Boston, MA: Shambhala.

Wikipedia Foundation, Inc. (2006). Sleep paralysis. Retrieved May 1, 2006, from http:// en.wikipedia.org/wiki/Sleep_paralysis

Zhi-ying, F., and Jian-xun, L. (1992). Near-death experiences among survivors of the 1976 Tangshan earthquake. Journal of Near-Death Studies, 11, 39-48.

Zubin, J., and Spring, B. (1977). Vulnerability: A new view on schizophrenia. Journal of Abnormal Psychology, 96, 103-126. 